\title{
An Effective Longitudinal Space-Charge Impedance Model for Beams with Non-uniform and Non-axissymmetric Transverse Density
}

\author{
Marco Venturini* \\ Lawrence Berkeley National Laboratory, University of California, Berkeley, California, 94720
}

(Dated: July 10, 2007)

Use of a one-dimensional model of longitudinal space-charge (SC) impedance has been proposed $[1,2]$ for studying the microbunching instability in single-pass delivery systems relevant for the next generation of FELs. For beams with uniform transverse density and circular cross-section of radius $r_{b}$ the SC impedance can be expressed in a handy analytical form, making this model particularly convenient. In this report we show how with an appropriate choice of $r_{b}$ one can use this as an effective-beam model to approximate beams with non-axissymmetric and non-uniform transverse densities.

PACS numbers: 29.27.Bd, 41.60.Ap

\section{INTRODUCTION}

Consider a single electron at $\left(x^{\prime}, y^{\prime}, z^{\prime}\right)$ moving along the $z$ axis at relativistic velocity with relativistic factor $\gamma$. The longitudinal electric field as seen in the lab frame is [3]

$$
E_{z}(x, y, z)=\frac{e}{4 \pi \varepsilon_{0}} \frac{\left(z-z^{\prime}\right) \gamma}{\left[\left(x-x^{\prime}\right)^{2}+\left(y-y^{\prime}\right)^{2}+\left(z-z^{\prime}\right)^{2} \gamma^{2}\right]^{3 / 2}}
$$

Similarly, a distribution of electrons with density $\rho_{\perp}(x, y) \lambda e^{i k z}$ where $\lambda$ is the longitudinal particle density and $\rho_{\perp}(x, y)$ the transverse density (normalized to unity $\int \rho_{\perp}\left(x^{\prime}, y^{\prime}\right) d x^{\prime} d y^{\prime}=1$ ), will generate a longitudinal electric field

$$
\begin{aligned}
E_{z}(x, y, z) & =\frac{e \lambda \gamma}{4 \pi \varepsilon_{0}} \int \frac{\left(z-z^{\prime}\right) \rho_{\perp}\left(x^{\prime}, y^{\prime}\right) e^{i k z^{\prime}} d x^{\prime} d y^{\prime} d z^{\prime}}{\left[\left(x-x^{\prime}\right)^{2}+\left(y-y^{\prime}\right)^{2}+\left(z-z^{\prime}\right)^{2} \gamma^{2}\right]^{3 / 2}} \\
& =\frac{e \lambda \gamma}{4 \pi \varepsilon_{0}} e^{i k z} \int \frac{\zeta \rho_{\perp}\left(x^{\prime}, y^{\prime}\right) e^{-i k \zeta} d x^{\prime} d y^{\prime} d \zeta}{\left[\left(x-x^{\prime}\right)^{2}+\left(y-y^{\prime}\right)^{2}+\zeta^{2} \gamma^{2}\right]^{3 / 2}} .
\end{aligned}
$$

If we set

$$
I_{k}=-\gamma \int \frac{\zeta \rho_{\perp}\left(x^{\prime}, y^{\prime}\right) e^{-i k \zeta} d x^{\prime} d y^{\prime} d \zeta}{\left[\left(x-x^{\prime}\right)^{2}+\left(y-y^{\prime}\right)^{2}+\zeta^{2} \gamma^{2}\right]^{3 / 2}}
$$

we have

$$
E_{z}=-\frac{e \lambda}{4 \pi \varepsilon_{0}} e^{i k z} I_{k}
$$

The longitudinal space-charge (SC) impedance (per unit length) for wave-number $k$ is defined as

$$
\hat{Z}(k)=\frac{1}{4 \pi c \varepsilon_{0}} I_{k}=\frac{Z_{0}}{4 \pi} I_{k},
$$

where $Z_{0}=120 \pi \Omega$ is the vacuum impedance.

\footnotetext{
*mventurini@lbl.gov
} 


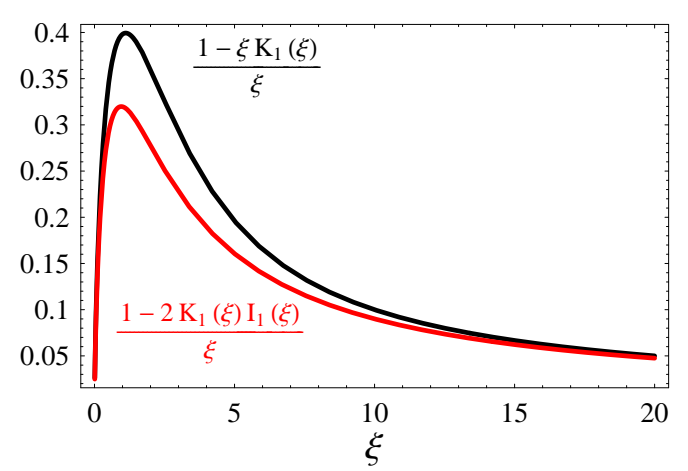

FIG. 1: The black curve is proportional to the SC impedance relative to the on-axis longitudinal electric field for a uniform beam with circular cross-section, see Eq. (8). The red curve is proportional the SC impedance averaged over the transverse density of the same beam, see Eq. (12). The variable $\xi$ is defined as $\xi=k r_{b} / \gamma$.

\section{TRANSVERSELY UNIFORM BEAM WITH CIRCULAR CROSS SECTION}

The transverse density is defined as $\rho_{\perp}(x, y)=1 / \pi r_{b}^{2}$ for $x^{2}+y^{2} \leq r_{b}^{2}$ and $\rho_{\perp}(x, y)=0$ for $x^{2}+y^{2}>r_{b}^{2}$. With a change to cylindrical coordinates the integral $I_{k}$ reads

$$
\begin{aligned}
I_{k} & =-\frac{2 \gamma}{r_{b}^{2}} \int_{0}^{r_{b}} r d r \int_{-\infty}^{\infty} d \zeta \frac{\zeta e^{-i k \zeta}}{\left(r^{2}+\zeta^{2} \gamma^{2}\right)^{3 / 2}} \\
& =-\frac{2}{\gamma r_{b}^{2}} \int_{-\infty}^{\infty} \frac{t d t}{\left(1+t^{2}\right)^{3 / 2}} \int_{0}^{r_{b}} d r e^{-i k t r / \gamma}=-\frac{2 i}{k r_{b}^{2}} \int_{-\infty}^{\infty} \frac{e^{-i t \xi}-1}{\left(1+t^{2}\right)^{3 / 2}} d t,
\end{aligned}
$$

where we have defined $\xi=k r_{b} / \gamma$ and have set the observation point on the axis $x=y=0$. From the table of integrals, $\int_{-\infty}^{\infty} d t /\left(1+t^{2}\right)^{3 / 2}=2$ and

$$
\int_{-\infty}^{\infty} \frac{e^{-i t \xi}}{\left[1+t^{2}\right]^{3 / 2}} d t=2 \xi K_{1}(\xi)
$$

where $K_{1}$ is Bessel function $K_{1}$. We have

$$
I_{k}=i \frac{4}{k r_{b}^{2}}\left[1-\xi K_{1}(\xi)\right]=\frac{4 i}{\gamma r_{b}} \frac{1-\xi K_{1}(\xi)}{\xi}
$$

in agreement with the expression reported in [4].

For $\xi \rightarrow \infty, K_{1}(\xi)$ decreases exponentially while for small $\xi$ we have $K_{1}(\xi) \simeq 1 / \xi+\xi\left[\log (\xi / 2)+\gamma_{E}-1 / 2\right] / 2$, where $\gamma_{E}$ is the Euler constant $\simeq 0.577$ Therefore we have the following limiting forms:

$$
\begin{aligned}
I_{k}(\xi \rightarrow \infty) & =i \frac{4}{k r_{b}^{2}}, \\
I_{k}(\xi \rightarrow 0) & =-i \frac{k}{\gamma^{2}}\left[2 \log (\xi)+2 \gamma_{E}-2 \log (2)-1\right] \simeq-i \frac{2 k}{\gamma^{2}} \log \left(\frac{k r_{b}}{1.85 \gamma}\right),
\end{aligned}
$$

where $2 \gamma_{E}-2 \log (2)-1 \simeq-1.23$

If the observation point is off-axis at a distance $r$ from the center the expression (8) for $I_{k}$ should be replaced [4] by

$$
I_{k}(r)=i \frac{4}{\gamma r_{b}} \frac{1}{\xi}\left[1-\xi K_{1}(\xi) I_{1}(k r / \gamma)\right]
$$

where $I_{1}$ is also a Bessel function.

It is interesting to compare $I_{k}$ on-axis (8) with the average of (11) over the transverse density:

$$
\left\langle I_{k}\right\rangle_{r}=\frac{\int_{0}^{r_{b}} I_{k}(r) \rho_{\perp}(r) r d r}{\int_{0}^{r_{b}} \rho_{\perp}(r) r d r}=\frac{4 i}{\gamma r_{b}} \frac{1-2 K_{1}(\xi) I_{1}(\xi)}{\xi} .
$$




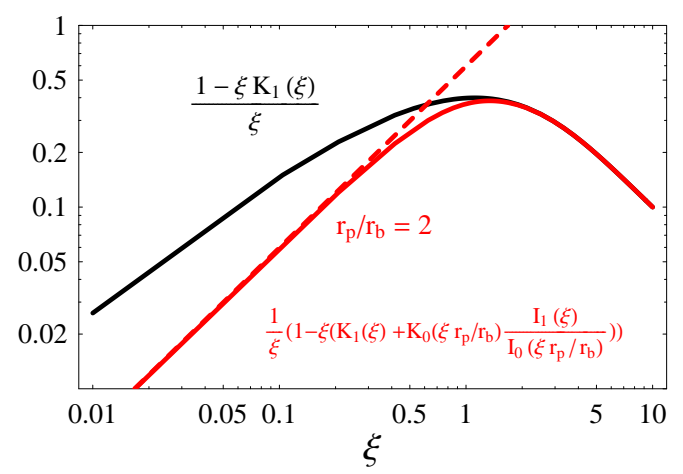

FIG. 2: The two solid line curves are proportional (with same proportionality factor) to the on-axis electric field with open boundary (black curve) and in the presence of a round perfectly conducting pipe at $r=r_{p}$ (red curve) with ratio $r_{p} / r_{b}=2$. The dashed line is the asymptotic expression $\xi\left[2 \log \left(r_{p} / r_{b}\right)+1\right] / 4$.

Because $I_{1}(\xi) \simeq \xi / 2$ for small $\xi$ and $I_{1}(\xi)$ decreases exponentially for large $\xi$, the limiting behavior of (12) at high and small frequencies is the same as (9) and (10). At intermediate frequencies, however, the averaged longitudinal field is noticeable smaller (red curve in Fig. 1).

In the presence of a perfectly conducting pipe of radius $r_{p}$ concentric with the charge distribution the expression (8) for $I_{k}$ should be replaced by

$$
I_{k}=i \frac{4}{\gamma r_{b}} \frac{1}{\xi}\left[1-\xi\left(K_{1}(\xi)+K_{0}\left(\xi r_{p} / r_{b}\right) \frac{I_{1}(\xi)}{I_{0}\left(\xi r_{p} / r_{b}\right)}\right)\right]
$$

The high frequency limit $\xi \rightarrow \infty$ is the same as $(9)$ as both $K_{0}\left(\xi r_{p} / r_{b}\right)$ and the ratio $I_{1}(\xi) / I_{0}\left(\xi r_{p} / r_{b}\right)$ decrease exponentially with $\xi$, whereas

$$
I_{k}(\xi \rightarrow 0)=i \frac{4}{\gamma r_{b}} \frac{\xi}{4}\left[2 \log \left(\frac{r_{p}}{r_{b}}\right)+1\right],
$$

having used $K_{0}(\xi)=-\left[\gamma_{E}+\log (\xi / 2)\right]+\mathcal{O}\left(\xi^{2}\right), I_{1}(\xi) \simeq \xi / 2$, and $I_{0}(\xi) \simeq 1$ for $\xi \rightarrow 0$. The effect of the boundary becomes significant at low frequencies below $\xi \simeq r_{b} / r_{p}$ or wavelengths $\lambda>2 \pi r_{p} \gamma$. See Fig. 2 .

\section{AXIS-SYMMETRIC BEAM WITH TRANSVERSE GAUSSIAN DENSITY.}

The transverse density is defined as $\rho_{\perp}(x, y)=e^{-\left(x^{2}+y^{2}\right) / 2 \sigma^{2}} / 2 \pi \sigma^{2}$. After changing to cylindrical coordinates we have

$$
I_{k}=i \frac{\xi}{\gamma \sigma} \sqrt{\frac{\pi}{2}} \int_{-\infty}^{\infty} \frac{t e^{-t^{2} / 2} \operatorname{erfi}(t / \sqrt{2}) d t}{\left(\xi^{2}+t^{2}\right)^{3 / 2}}
$$

where the erfi( $t)$ function is defined as erfi $(t)=(2 / \sqrt{\pi}) \int_{0}^{t} e^{x^{2}} d x$, and $\xi=k \sigma / \gamma$.

To study the limiting form of the above expression at high and low frequencies consider the function

$$
F(s)=\sqrt{\frac{\pi}{2}} \int_{-\infty}^{\infty} \frac{t e^{-t^{2} / 2} \operatorname{erfi}(t / \sqrt{2}) d t}{\left(s+t^{2}\right)^{3 / 2}} .
$$

The function in the numerator of the integrand of (16) is zero at $t=0$, and for large $t$ tends asymptotically to $\sqrt{2 / \pi}$. In the limit of large $s$ most of the contribution from the integrand comes for large $t$. We can then approximate

$$
F(s) \simeq \sqrt{\frac{\pi}{2}} \int_{-\infty}^{\infty} \frac{\sqrt{2 / \pi} d t}{\left(s+t^{2}\right)^{3 / 2}}=\frac{2}{s} .
$$




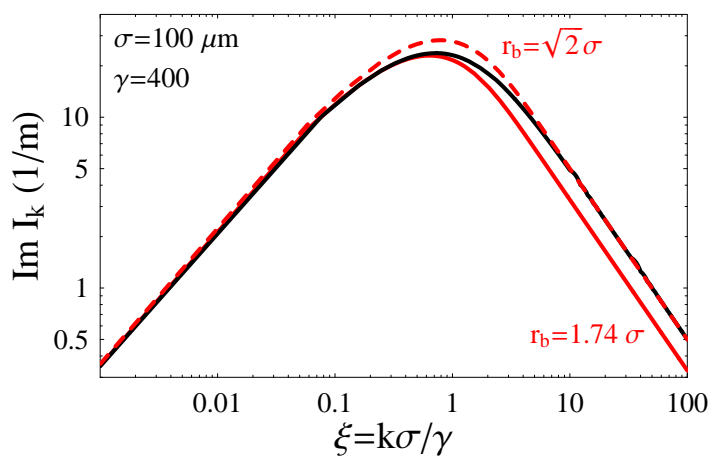

FIG. 3: The (absolute value of the) quantity $I_{k}$ as a function of $\xi=k \sigma / \gamma$ for an axis-symmetric gaussian beam with transverse rms sizes $\sigma$ [black curve, see Eq. (15)] is contrasted with that of a beam with uniform transverse density for two choices of the radius $r_{b}$ (red curves).

For small $s, F(s)$ diverges logarithmically, with $F(s)=A \log (s)+B$. The coefficient $A$ can be easily determined analytically. To this end we replace the numerator in the integrand of (16) with the first non-vanishing term of its Taylor expansion $t e^{-t^{2} / 2} \operatorname{erfi}(t / \sqrt{2}) \simeq \sqrt{2 / \pi} t^{2}$ :

$$
F(s) \simeq 2 \int_{0}^{\Lambda} \frac{t^{2} d t}{\left(s+t^{2}\right)^{3 / 2}}=\left.2\left[-\frac{t}{\sqrt{t^{2}+s}}+\log \left(t+\sqrt{t^{2}+s}\right)\right]\right|_{t=0} ^{t=\Lambda}=2\left[-\frac{\Lambda}{\sqrt{\Lambda^{2}+s}}+\log \frac{\Lambda+\sqrt{\Lambda^{2}+s}}{\sqrt{s}}\right]
$$

where $\Lambda$ is some cut-off. In the limit $s \rightarrow 0$ we read off the coefficient of $\log (s)$ to find $A=-1$. The coefficient $B$ can then be computed numerically as the difference $B=F(s)-A \log (s)$ for $s$ sufficiently small. We found $B \simeq 0.116$.

We can now write the limiting forms of in the high and low frequency limits

$$
\begin{aligned}
I_{k}(\xi \rightarrow \infty) & =i \frac{2}{\gamma \sigma \xi}=\frac{2 i}{\sigma^{2} k}, \\
I_{k}(\xi \rightarrow 0) & =-i \frac{\xi}{\gamma \sigma}[2 \log (\xi)-0.116]=-i \frac{2 k}{\gamma^{2}} \log \left(\frac{k \sigma}{1.06 \gamma}\right),
\end{aligned}
$$

By comparing (19) and (20) to (9) and (10) respectively we can define a uniform beam with transverse size $r_{b}$ 'equivalent' to a gaussian beam with $\mathrm{rms}$ size $\sigma$. In the high frequency limit $r_{b}^{\text {eqv }}=\sqrt{2} \sigma$, whereas in the low frequency limit $r_{b}^{\text {eqv }} \simeq(1.85 / 1.06) \sigma=1.74 \sigma$. A numerical evaluation of (15) shows that at intermediate frequencies $r_{b}^{\text {eqv }}$ falls in the interval $[\sqrt{2}, 1.74]$. See Fig. 3 .

\section{TRANSVERSALLY UNIFORM BEAM WITH ELLIPTICAL CROSS SECTION}

The transverse density is defined as $\rho_{\perp}\left(x^{\prime}, y^{\prime}\right)=1 / \pi a b$ for $(x / a)^{2}+(y / b)^{2} \leq 1$ and $\rho_{\perp}\left(x^{\prime}, y^{\prime}\right)=0$ outside the ellipse. For observation point on axis the integral can be reduced to

$$
I_{k}=\frac{i}{\pi k}\left[\frac{4 \pi}{a b}-\frac{2 k}{\gamma} \int_{0}^{2 \pi} \frac{K_{1}(k \alpha(\phi) / \gamma)}{\alpha(\phi)} d \phi\right]
$$

where $\alpha(\phi)=\sqrt{a^{2} \cos ^{2} \phi+b^{2} \sin ^{2} \phi}$

In the high frequency limit the contribution from the integral vanishes as the Bessel function $K_{1}$ decreases exponentially and the limiting expression for $I_{k}$ is easily found. At low frequency the calculation is slightly more involved but can be still be expressed in terms of elementary functions by making use of the limiting expression for $K_{1}$ for small argument and the integral $\int_{0}^{2 \pi} d t \log \left(1+c^{2} \sin ^{2} t\right)=4 \pi \log \left(\left[1+\sqrt{1+c^{2}}\right] / 2\right)$. The result is very similar to the expression obtained for round beams:

$$
\begin{aligned}
I_{k}(\xi \rightarrow \infty) & =i \frac{4}{k a b} \\
I_{k}(\xi \rightarrow 0) & =-i \frac{k}{\gamma^{2}}\left[2 \log \left(\frac{k(a+b)}{2 \gamma}\right)+2 \gamma_{E}-2 \log (2)-1\right] \simeq-i \frac{2 k}{\gamma^{2}} \log \left(\frac{k}{1.85 \gamma} \frac{a+b}{2}\right),
\end{aligned}
$$




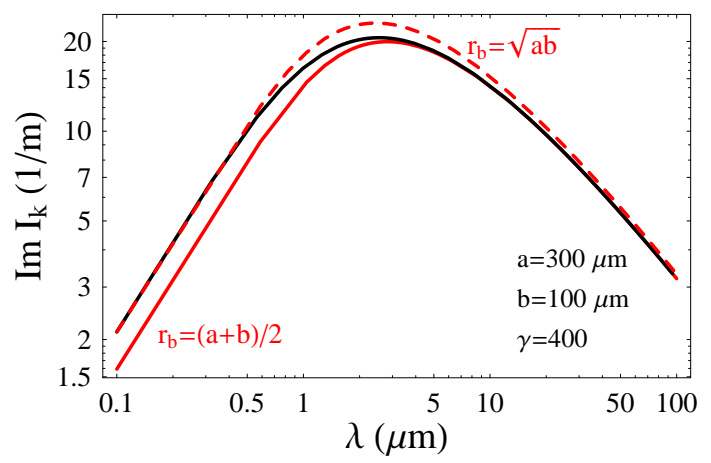

FIG. 4: The (absolute value of ) the quantity $I_{k}$ [Eq.(21)] as a function of wavelength $\lambda=2 \pi / k$ for a transversely uniform beam with elliptical cross section with axes $a$ and $b$ (black curve) compared with that of round, uniform beam $r_{b}=\sqrt{a b}$ (red dashed curve), and $r_{b}=(a+b) / 2$ (red solid line).

Again, we can define an 'equivalent' uniform, round beam by comparing (22) and (23) to (9) and (10) respectively. For high frequencies we find $r_{b}^{\text {eqv }}=\sqrt{a b}$. In the low-frequency limit $r_{b}^{\text {eqv }}=(a+b) / 2$. See Fig. 4 .

\section{TRANSVERSALLY GAUSSIAN BEAM WITH UNEQUAL RMS SIZES}

The transverse density in this case is defined as $\rho_{\perp}(x, y)=e^{-\left(x^{2} / 2 \sigma_{x}^{2}+y^{2} / 2 \sigma_{y}^{2}\right)} / 2 \pi \sigma_{x} \sigma_{y}$ and the expression $I_{k}$ can be reduced to

$$
I_{k}=i \frac{k}{2 \pi \gamma^{2}} \int_{0}^{2 \pi} d \phi F\left(\frac{k^{2} \alpha^{2}(\phi)}{\gamma^{2}}\right)
$$

where $\alpha(\phi)=\sqrt{\sigma_{x}^{2} \cos ^{2} \phi+\sigma_{y}^{2} \sin ^{2} \phi}$ and $F(s)$ was defined in (16). For large $s, F(s) \simeq 2 / s$ and therefore

$$
I_{k}(k \rightarrow \infty)=i \frac{k}{\pi \gamma^{2}} \frac{\gamma^{2}}{k^{2}} \int_{0}^{2 \pi} \frac{d \phi}{\alpha^{2}(\phi)}=i \frac{2}{k \sigma_{x} \sigma_{y}} .
$$

For small $s$ we use the approximation $F(s) \simeq-\log (s)+0.116$ as in Sec. III and obtain:

$$
I_{k}(\xi \rightarrow 0)=i \frac{k}{\gamma^{2}}\left[2 \log \left(\frac{\sigma_{x}+\sigma_{y}}{2} \frac{k}{\gamma}\right)-0.116\right]=i \frac{2 k}{\gamma^{2}} \log \left(\frac{\sigma_{x}+\sigma_{y}}{2} \frac{k}{1.06 \gamma}\right)
$$

\section{CONCLUSIONS}

A simple and convenient model to study longitudinal effects of space charge is that of a round beam with transverse uniform density. Most often, the beam is neither axis-symmetric or uniform, but we would still like use an 'equivalent' round and uniform beam model because of its simplicity. For gaussian beams with rms sizes $\sigma_{x}$ and $\sigma_{y}$, limiting expressions at small frequencies suggest that we could use an equivalent axis-symmetric uniform beam with radius $r_{b} \simeq 1.74\left(\sigma_{x}+\sigma_{y}\right) / 2$. This is in substantial agreement with the expression suggested in [1]. Numerical calculations show that this choice is reasonably accurate over a large spectrum of frequencies and moderate aspect-ratios. Significant deviations only begin to occur at high frequencies for $k\left(\sigma_{x}+\sigma_{y}\right) / 2 \gamma>1$. We should remark that the definition of equivalent beam is limited to consideration of the on-axis longitudinal electric field. One may argue that a more meaningful quantity is the longitudinal electric field averaged over the transverse beam density. For round beams we showed that this can be up to about $20 \%$ smaller than the on-axis field for a range of frequencies in the neighborhood of $k r_{b} / \gamma \simeq 1$. 
We are thankful to A. Zholents and M. Furman for useful discussions and suggestions.

[1] Z. Huang, M. Borland, P. Emma, J. Wu, C. Limborg, G. Stupakov, and J. Welch, Phys. Rev. ST Accel. Beams 7, 074401 (2004); the authors recommend using $r_{b}=1.7\left(\sigma_{x}+\sigma_{y}\right) / 2$.

[2] M. Venturini, R. Warnock, and A. Zholents, Phys. Rev. ST Accel. Beams 10, 054403 (2007).

[3] J.D. Jackson, Classical Electrodynamics, Wiley (1974).

[4] J. Rosenzweig, C. Pellegrini, L. Serafini, C. Ternieden, and G. Travish, Nucl. Instrum. Methods Phys. Res., Sect. A 393, 376 (1997) 
This document was prepared as an account of work sponsored by the United States Government. While this document is believed to contain correct information, neither the United States Government nor any agency thereof, nor The Regents of the University of California, nor any of their employees, makes any warranty, express or implied, or assumes any legal responsibility for the accuracy, completeness, or usefulness of any information, apparatus, product, or process disclosed, or represents that its use would not infringe privately owned rights. Reference herein to any specific commercial product, process, or service by its trade name, trademark, manufacturer, or otherwise, does not necessarily constitute or imply its endorsement, recommendation, or favoring by the United States Government or any agency thereof, or The Regents of the University of California. The views and opinions of authors expressed herein do not necessarily state or reflect those of the United States Government or any agency thereof, or The Regents of the University of California.

Ernest Orlando Lawrence Berkeley National Laboratory is an equal opportunity employer. 